\title{
[Sensitive Apparatus For Measurement of Electric Charges on Precipitation ${ }^{1}$
}

\author{
Charles A. Ratcliffe, M. Terry Dana and Edwin M. Sheen \\ Battelle-Northwest, Richland, Wash. \\ (Manuscript received 16 December 1968, in revised form 28 february 1969)
}

\begin{abstract}
An inductor ring device capable of measuring weak electric charges on precipitation was developed. The electronics of the transducer employs a virtual ground configuration for stability in operation and increased sensitivity and is housed in a case designed for effective field operation. Field experience in nonthunderstorm precipitation has shown the instrument to be capable of measuring charges as low as 2 femtocoulombs $\left(2 \times 10^{-15} \mathrm{C}\right)$. Charge distributions for raindrops were determined for precipitation characteristic of the Pacific Northwest and found to be primarily positive in polarity.
\end{abstract}

\section{Introduction}

In the past there has been considerable interest in the electric charges on raindrops and snowflakes as evidenced by the early work of Gunn (1957) and others with thunderstorm electrification. More recently, this laboratory has been interested in correlating precipitation particle charges with the efficiency of precipitation in the removal of particulate contaminants. The current instrument was specifically designed to measure the relatively weak electric charges associated with the nonthunderstorm precipitation common to the coastal areas of the Pacific Northwest.

\section{Instrument development}

The transducer is the key to any workable system. The authors' first attempts used barrel-like enclosures with "inductor rings" mounted inside. This terminology derives from the induction of charge in the ring as the particle approaches and falls through the ring without contact. The barrel enclosure was so large that wind effects in the field caused a large fraction of the particles to hit the inductor rings, and tilting of the barrel was

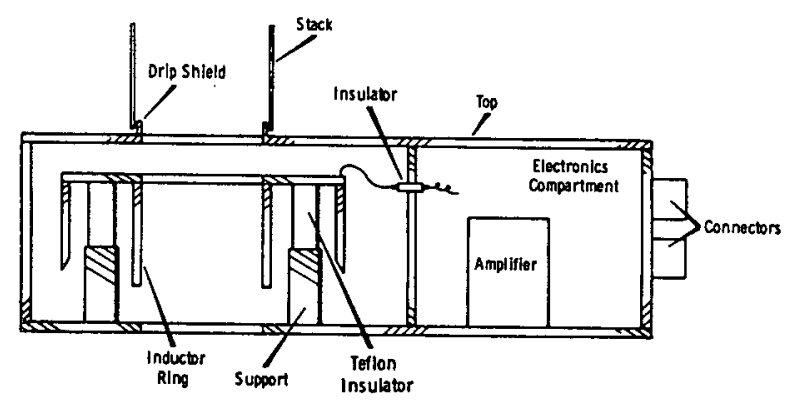

FIG. 1. Cross section of transducer.

${ }^{1}$ This work was performed under Atomic Energy Commission Contract No. AT (45-1)-1830. not effective. Wetting of the insulators (which separate the inductor rings from the case) was a problem. Also annoying at times were the undesirable effects of microphonics. The latter problem is believed to be due to relatively flimsy construction (long insulators) and to the minute changes in the capacitance that introduced extraneous signals.

A much sturdier transducer was built from 0.25 -inch aluminum alloy parts bolted together. This unit, shown nearly to scale in Fig. 1, has an insulator arrangement which is nearly impossible to wet, superior microphonic characteristics, and improved geometry for induction. It is about 12 inches long, 7 inches wide and 4 inches high. The stack, drip shield, inductor ring and support are cylindrical, machined components. As can be seen, the inductor ring is shaped so as to shield the Teflon insulators from droplet splashes. As discussed later in the paper, the inductor ring operates electrically at "virtual ground" or essentially zero potential with respect to the rest of the transducer enclosure. This improvement reduces both the voltage stress on the insulators and any errors produced by charge leakage across the insulators.

The transducer using Teflon insulators was tested in an environmental chamber and was found to be immune to any conditions of temperature and humidity which would be encountered in the field. Teflon insulators were found to be definitely superior to cleaned, waxed steatite insulators.

The amount of charge induced in the ring by a given raindrop charge will be nearly proportional to the solid angle subtended by the inductor ring when the drop is inside. This conclusion may be reached by considering the lines of electric feld emanating from the drop. If the drop goes through, the induced charge will vary with the aspect ratio (height to diameter) of the ring and with the radial position of the path with respect to the center line of the ring. An attempt was made to reduce 
the radial variation by making the aspect ratio $\sim 1$, where in our previous models it had been on the order of 0.1. A compromise is involved, of course, as a large aspect ratio ring is more likely to intercept drops due to variations in the wind velocity in the field. If the drop hits the ring, all of the charge is transferred to the ring, it being held at virtual ground potential by the electronic system. The system utilized a ring so that a particle would be allowed to pass on to some other future device for correlative studies.

The electronic portion of the device consists of an amplifier, power supply and recorder. The amplifier may be operated as a voltage follower, as has been used in earlier devices recorded in the literature, or in a virtual ground configuration. With the amplifier connected as a follower, the system sensitivity depends on the total capacitance of ring and wiring to ground, resulting in lower sensitivity. The possibility of capacitance change due to vibration or position change of the connecting wire and variation of amplifier input capacitance with time and between units exists as well. Minimum attainable capacitance is of the order of $10-20 \mathrm{pF}$ with this mode.

Operation with a virtual ground is different in that the charge is induced in a member which is held at ground potential by means of the amplifier and a feedback loop, thus greatly reducing possible amplitude effects. Charge sensitivity under this arrangement is determined by a feedback capacitor which may be of the order of $1-2 \mathrm{pF}$, resulting in about an order of magnitude increase in sensitivity.

With regard to microphonics or spurious outputs due to vibration of the transducer (e.g., by raindrops hitting it), consider the following two cases. For the voltage follower or historical case, assume a transducer capacitance $C$ with variation $C$, an input offset voltage $V_{0}$, and gain $A$ to give a particular sensitivity in volts per coulomb. The voltage output for this case is

$$
V_{1}=A\left(\frac{Q+Q_{0}}{C}-K_{1}\right)
$$

where $Q$ is the input signal charge and $K_{1}$ a constant. The change in output voltage for a perturbation of $C$ (assuming no signal) is

$$
d V_{1} / d C=-A Q_{0} / C^{2}=-A V_{0} / C .
$$

For the virtual ground case obtained in this work, with $C$, variation $C$, offset $V_{0}$, and amplifier gain very large compared to 1 , the signal voltage is

$$
V_{2}=\frac{V_{0} C+V_{0} C_{f}-Q}{C_{f}}-K_{2}
$$

where $C_{f}$ is the feedback capacitance and $K_{2}$ a constant. The feedback capacitance, about $0.1 \mathrm{C}$, is physically

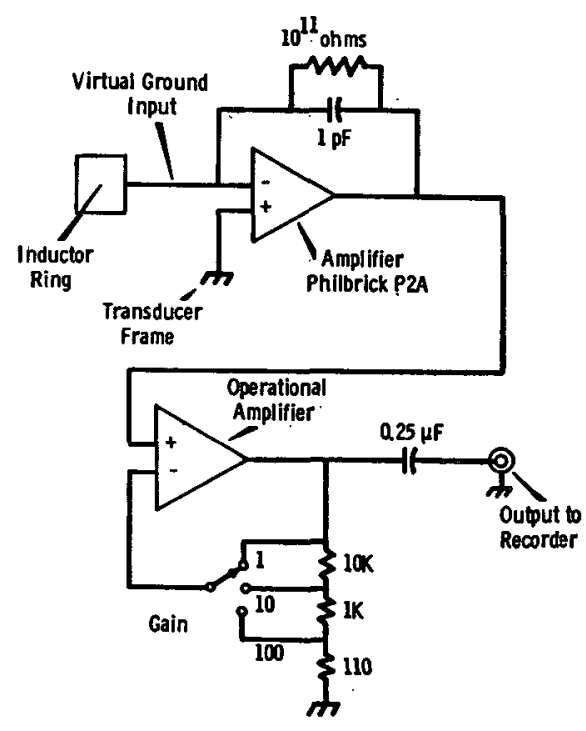

Fig. 2. Electronic apparatus.

chosen and mounted so as to be constant. Here we find

$$
d V_{2} / d C=V_{0} / C_{f}
$$

For equal sensitivities $(V / Q)$ of the two systems, we obtain

$$
A / C=1 / C_{f} \quad \text { or } \quad A=C / C_{f} .
$$

Thus (2) becomes

$$
d V_{1} / d C=-C V_{0} / C C_{f}=-V_{0} / C_{f} .
$$

Now the $V_{0}$ for the follower case results from grid current and other leakage currents flowing in the input resistor and can hardly be reduced below $10^{-13}$ ampere $\times 10^{11} \mathrm{ohms}$ or $0.01 \mathrm{~V}$, with possibly $0.005 \mathrm{~V}$ as a lower limit. For the virtual ground case, the operational amplifier sets $V_{0}$, its amplitude being of the order of $0.0005 \mathrm{~V}$ (but no worse than $0.001 \mathrm{~V}$ ), thus gaining about a tenfold improvement. For a $\Delta C$ of only $\frac{1}{2} \mathrm{pF}$, the microphonic noise of the follower case will be

$$
\Delta V_{1}=-(0.01 \mathrm{~V})\left(0.5 \times 10^{-12}\right) /\left(1.5 \times 10^{-12}\right),
$$

or about $3.3 \mathrm{mV}$; this is equivalent to a bonafide observable charge of $\left(3.3 \times 10^{-3}\right.$ volt $)\left(1.6 \times 10^{-12}\right.$ coulombs per volt $) \approx 5.0$ femtocoulombs $\left(5 \times 10^{-15} \mathrm{C}\right)$.

The system relaxation time or time constant is determined by the feedback capacitance in parallel with whatever resistance exists or is placed there by the designer. Too short a time constant (small resistance) results in a small signal proportional to the arriving current, and too long a time constant often results in system paralysis when the inductor ring is struck by an incoming particle. A time constant of about $150 \mathrm{msec}$ appeared to provide a good compromise. This required a resistance of $10^{11} \Omega$. The present apparatus (Fig. 2) uses a Philbrick Researches Model P2A operational 


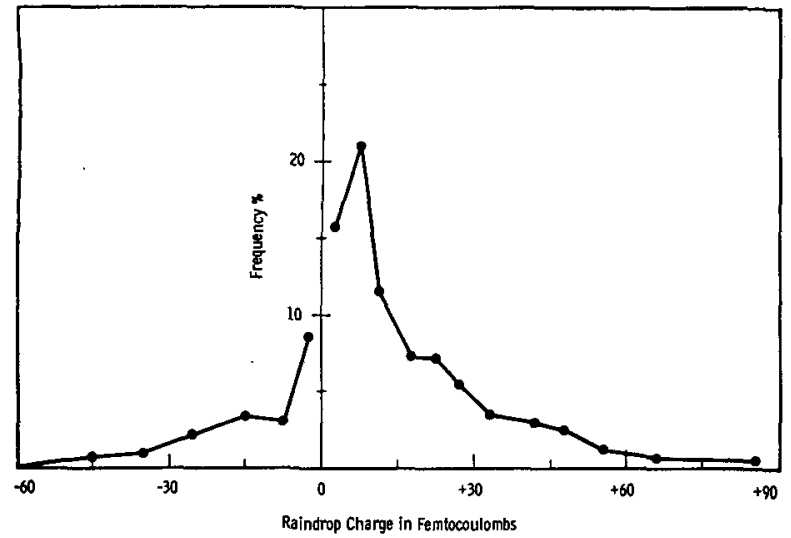

FIG. 3. Charge distribution for Quillayute, 28 March 1968.

amplifier, chosen for its very high input impedance and gain. A similar amplifier is the Analog Devices, Inc., Model 301. As in any electronic system, the internal noise determines the threshold of sensitivity. The noise level for this device in our system is about $10^{-15} \mathrm{C}$.

A post amplifier is incorporated into the system to increase the effective sensitivity of the system by a selectable factor of 1,10 or 100 . The amplifier is coupled to the recorder by a capacitor to remove variations in quiescent output voltage. The field power supply provides regulated outputs of plus and minus $15 \mathrm{~V}$ with respect to circuit common. The recorder presently employed is a Brush Mark II, Model RD 2522-20 dual channel unit with electric writing to obviate pen inking difficulties. It has an input impedance of $5 \mathrm{M} \Omega$ and maximum sensitivity of $10 \mathrm{mV} \mathrm{mm} \mathrm{mm}^{-1}$.

Laboratory calibration with water drops artificially charged to known values indicated a response, which is independent of radial position to within about $3 \%$, of $1.61 \mathrm{pC}$ per volt output for passage of the drop through the inductor ring without contact, and $1.45 \mathrm{pC}$ per volt for the case where the drop hits the ring. The latter pulse shape is easily identifiable on the record by its exponential decay; thus, the appropriate calibration factor may be applied to each type of pulse on the recording.

\section{Field Experience}

Field observations of precipitation from stratus and stratocumulus clouds were made in several locations in Washington State. Other data collected concurrently included precipitation intensity, raindrop size, and surface meteorological conditions. Measurements at the Quillayute Weather Bureau Station, on the Pacific Coast of the Olympic Peninsula, were made in conjunction with precipitation scavenging field experiments.

The transducer, mounted on a camera tripod, and power supply were located at sufficient distance from the recorder and other electrical equipment to limit the effect of power line noise. A steel plate below the inducing ring further acted as an extraneous field shield and was slanted sufficiently to prevent raindrops from splashing back into the ring.

Typical field sensitivity has allowed a charge magnitude of $3 \mathrm{fC}$ to be read with ease from the record, but observations of charges as low as $2 \mathrm{fC}$ (near the assumed internal noise level) were noted. Power line noise was rarely a problem, even when the transducer was within $20 \mathrm{ft}$ of the trailer housing the recorder. In all sampling periods, both channels of the recorder were used at different sensitivities to capture the full range of magnitudes of charge in a given rain. Often the ratio of highest to lowest was 250 or more. In some of the heavy rain samples a few small pulses may have been missed while the system recovered from a large pulse.

Sample periods of several minutes duration each were selected from the record and plotted as statistical distributions. The distribution in Fig. 3 represents a sample taken with the best measurement sensitivity as yet attained. The abcissa is raindrop charge in femtocoulombs and the ordinate is the number frequency of charge values in normalized charge intervals. Table 1 lists pertinent charge and precipitation intensity data for several representative sample periods. In both Fig. 3 and Table 1 it must be noted that the number of raindrops having charge magnitudes below the measurement capabilities of the instrument was not determined and thus not included in the averaging. The contribution to the charge data from double (simultaneous) pulses is considered negligible due to the instrument's fast response and recovery; in addition, very dense records. (greater than 2 or 3 pulses $\mathrm{sec}^{-1}$ ) are not generally analyzed.

Precipitation intensity was measured by means of a recording weighing raingage developed by one of the authors. The resolution of the gage is about $0.06 \mathrm{~mm}$ of rainfall, and allowed determinations of rainfall rate over time intervals of 1-2 min. Upper level meteorological data was minimal during the periods listed in Table 1 , but it is safely assumed that in each case the rain resulted from melted solid hydrometeors. No measurement of atmospheric electrical conductivities or electric field were made.

The sampling efficiency of the transducer has not been quantitatively determined for field operation. Obviously, determining factors are the aerodynamic properties of the transducer as well as wind and turbulence around the induction ring during each measurement period. There are, as well, undetermined and variable numbers of raindrops whose charges may be too small to be measured. Windy conditions, resulting in nonvertical fall paths of raindrops, do reduce the sampling efficiency. In the limited periods of data collection under these conditions, the inductor ring axis was inclined into the wind to the position of greatest number of pulses recorded per unit time. 
TABLE 1. Raindrop charge data reduced from typical field runs.

\begin{tabular}{|c|c|c|c|c|c|c|c|c|}
\hline \multirow[t]{2}{*}{ Location } & \multirow[t]{2}{*}{ Date } & \multicolumn{2}{|c|}{$\begin{array}{l}\text { Number of } \\
\text { measurable } \\
\text { charges }\end{array}$} & \multicolumn{2}{|c|}{$\begin{array}{l}\text { Average } \\
\text { charge } \\
\text { (fC) }\end{array}$} & \multirow[t]{2}{*}{$\begin{array}{l}\text { Average } \\
\text { net } \\
\text { charge } \\
\text { (fC) }\end{array}$} & \multirow[t]{2}{*}{ 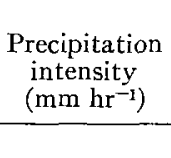 } & \multirow[t]{2}{*}{$\begin{array}{l}\text { Wind } \\
\text { speed } \\
\text { (mph) }\end{array}$} \\
\hline & & + & - & + & - & & & \\
\hline Hanford, Wash. & $11-8-67$ & 72 & 0 & 144 & 0 & +144 & - & Calm \\
\hline Hanford, Wash. & $11-8-67$ & 178 & 59 & 95 & 140 & +37 & - & 5 \\
\hline Skykomish, Wash. & $1-9-68$ & 148 & 63 & 22 & 19 & +10 & - & Calm \\
\hline Skykomish, Wash. & $2-20-68$ & 203 & 14 & 68 & 39 & +61 & 1.5 & Calm \\
\hline Skykomish, Wash. & $2-21-68$ & 76 & 141 & 537 & 708 & -272 & 3.9 & Calm \\
\hline Skykomish, Wash. & $2-22-68$ & 166 & 49 & 295 & 169 & +190 & 11.5 & Calm \\
\hline Skykomish, Wash. & $2-22-68$ & 102 & 90 & 65 & 71 & +4 & 1.1 & Calm \\
\hline Quillayute, Wash. & $3-26-68$ & 527 & 134 & 1.58 & 112 & +103 & 9.1 & 9 \\
\hline Quillayute, Wash. & $3-28-68$ & 426 & 36 & 93 & 21 & +91 & 1.9 & 4 \\
\hline Quillayute, Wash. & $3-28-68 *$ & 144 & 42 & 22 & 17 & +13 & 2.2 & Calm \\
\hline
\end{tabular}

* Illustrated in Fig. 3.

The general shape of the distribution in Fig. 3 is typical of all the collected data for rainfall-approximately Gaussian with a peak near zero charge. With few exceptions, the distributions for nonthunderstorm raindrops have been observed to be peaked on the positive side of zero charge in agreement with the finds of Gunn $(1955,1957)$.

A few samples of snowflake charges were collected at Skykomish, Wash., in the Cascade Mountains. A definite interpretation of the pulses, which are of odd shapes-presumably due to varying fall velocities and geometric factors-has not been determined. A qualitative examination of these limited data indicates, however, that the predominant polarity was negative.

\section{Conclusion}

An instrument has been devised for measurement of electric charges on precipitation particles. Its anticipated superior sensitivity has been verified by field experience.

Acknowledgments. The authors thank N. C. Hoitink, J. L. Stringer and R. C. Weddle for their contributions in electronics and temperature-humidity testing.

\section{REFERENCES}

Gunn, R., 1955: The systematic electrification of mist and light rain in the lower atmosphere. $J$. Geophys. Res., 60, 23-27. $\longrightarrow, 1957$ : The electrification of precipitation and thunderstorms. Proc. IRI, 45, 1331-1358. 